\title{
Comportamento do par tribológico grafite-aço analisado a partir da influência do plano basal
}

\section{Behavior of the tribological pair graphite-steel analyzed from the influence of the basal plane}

\author{
Celso Oliveira ${ }^{1,2}$, Maria Aparecida Miranda de Souza ${ }^{2}$, Luiz Claudio Pardini²
}

\section{RESUMO}

O presente trabalho avalia o comportamento de pares tribológicos formados por grafites extrudados policristalinos e aço 4340. Os ensaios foram efetuados com o intuito de correlacionar a distribuição dos planos basais do grafite, identificada por meio de análises de microscopia óptica com luz polarizada, em relação ao coeficiente de fricção resultante, determinado utilizandose um tribômetro de pino sobre disco. Os materiais estudados mostraram aumento nos valores do coeficiente de fricção com o aumento do domínio do plano basal perpendicular à superfície de contato do par tribológico.

Palavras-chave: Coeficiente de fricção; Plano basal; Grafite.

\begin{abstract}
The objective of this study was to evaluate the tribological behavior of a couple formed by polycrystalline extruded graphite and 4340 steel. The tests were carried out by means of a pin on disc tribometer to determine the correlation of distribution of basal graphite planes and the resulting coefficient of friction by analyzing the polarized light using an optical microscopy. The studied materials presented increasing friction coefficient values with the increasing area of basal plane normal to the contact surface of the tribological couple.
\end{abstract}

Keywords: Coefficient of friction; Basal plane; Graphite. 


\section{INTRODUÇÃO}

\section{Considerações Gerais}

A tribologia une os campos da mecânica, física e química, assim como materiais, aos conhecimentos de lubrificação, atrito e desgaste para predizer o comportamento de sistemas físicos. Os principais temas do estudo da tribologia são o desgaste e o coeficiente de fricção $(\mathrm{COF})^{(1)}$. Esses fenômenos são influenciados por uma variedade de fatores, sendo que os principais são a temperatura, as condições ambientais, os gases ou líquidos presentes e a formação de filmes superficiais durante o processo de desgaste.

A influência do comportamento tribológico entre os materiais abrange 4 partes estruturais: a interação entre o par tribológico (sistema tribológico) em estudo, as condições ambientais, a lubrificação e as condições de operação. Dependendo do tipo de sistema tribológico (rolamento, freio, embreagem etc.) e das condições de operação (força normal, velocidade, temperatura, tempo de operação e distância de operação), vários processos de atrito e desgaste podem ocorrer. $\mathrm{O}$ esquema de interações desses fatores está demonstrado na Fig. $1^{(2)}$.

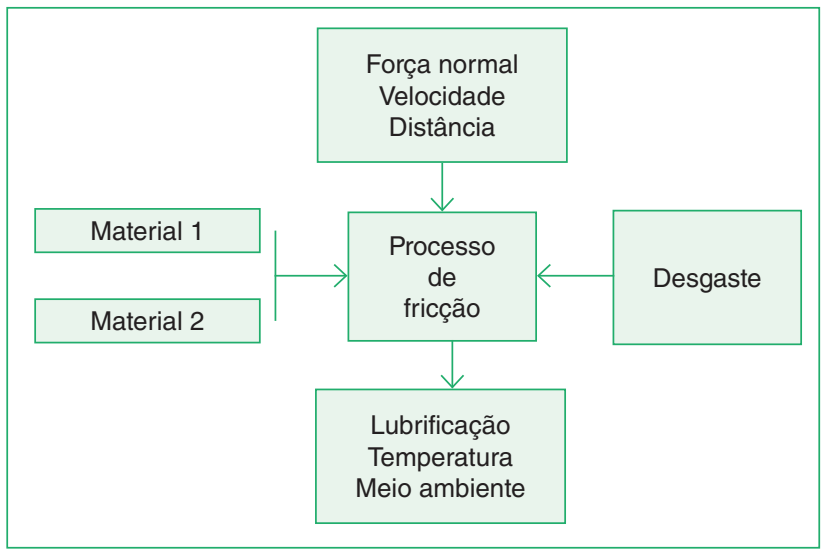

Figura 1: Representação esquemática dos fatores de influência no sistema tribológico e sua resposta no processo de fricção e desgaste ${ }^{(2)}$

Os processos de desgaste podem ser lubrificados ou secos e de 2 ou 3 corpos, conforme mostrado na Fig. 2. Diferentemente do processo de interação de 2 corpos, no processo de 3 corpos, partículas presentes no sistema tribológico, debris, ficam aprisionadas entre 2 superfícies, gerando um sistema tribológico de 3 corpos. Além disso, essas partículas podem ser oriundas da interação de um sistema de $2 \operatorname{corpos}^{(3)}$.

Materiais carbonosos, tais como o grafite, são de grande interesse para a engenharia devido às suas propriedades térmicas, elétricas e lubrificantes, o que os torna versáteis para aplicações nas áreas metalúrgica, de materiais sensíveis, de transporte, entre outras.

A Fig. 3 mostra a célula da unidade básica estrutural do cristal de grafite. Em condições ambientais normais de umidade relativa
(UR) e temperatura, $\sim 50 \%$ UR e $23^{\circ} \mathrm{C}$, o COF do grafite é baixo devido aos planos basais possuírem orientações praticamente paralelas à superfície; esses planos possuem baixa energia superficial quando expostos uns aos outros.

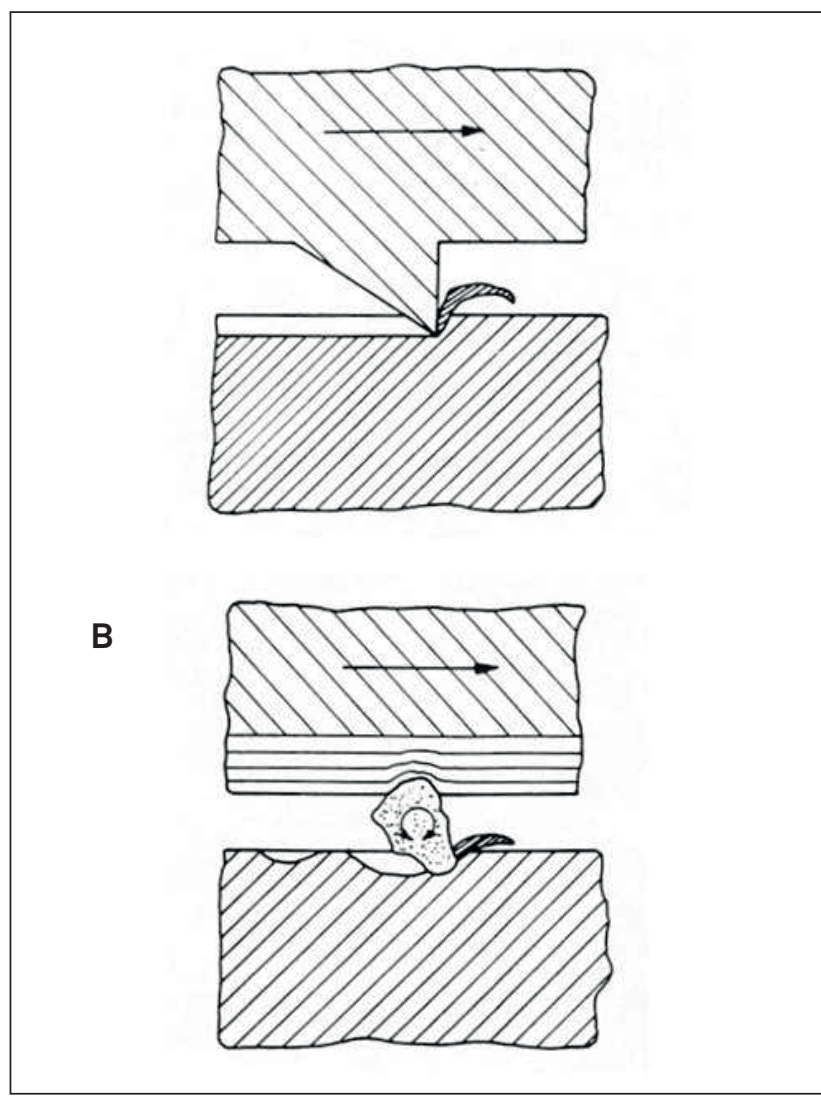

Figura 2: Esquema de desgaste: A) a 2 corpos, B) a $3 \operatorname{corpos}^{(1)}$.

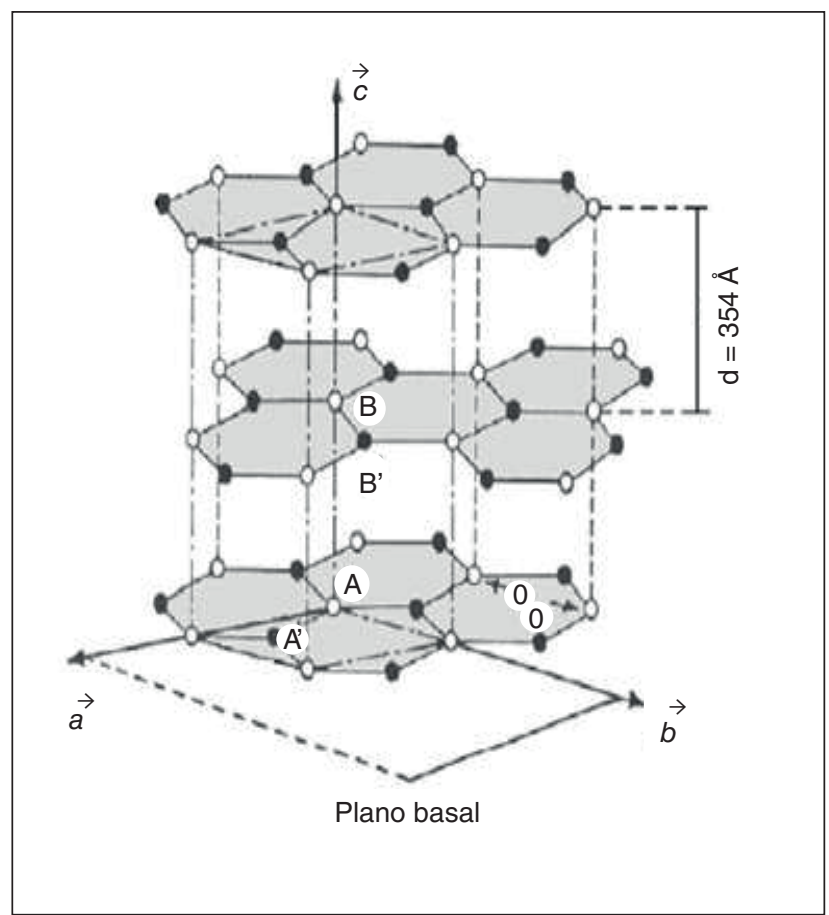

Figura 3: Estrutura hexagonal de um monocristal de grafite $^{(4)}$. 
Quandoé iniciado dano ao plano basal, regiões de alta energia são expostas, com maior capacidade de adesão. A fricção e o desgaste são reduzidos se essas regiões forem neutralizadas pela ação de gases ativos, como, por exemplo, a água adsorvida. Essa ação lubrificante da água pode ser explicada como resultado da interação com as partículas residuais formadas no processo de desgaste, os debris ${ }^{(5,6)}$.

Tal efeito diminui a interação entre as faces de contato, por meio da formação de um filme lubrificante, reduzindo a adesão entre elas, por neutralização das ligações químicas rompidas durante o processo de atrito, provocando, consequentemente, um decréscimo no $\mathrm{COF}^{(5,6)}$.

A formação do filme também está ligada diretamente à plasticidade dos debris formados, o que indiretamente pode ser indicado pelo módulo elástico do material, uma vez que o COF está associado à resistência ao cisalhamento interfacial e à dureza da superfície ${ }^{(7)}$. O comportamento tribológico do grafite tem sido foco de muitos trabalhos que estudaram seu desempenho como lubrificante em diferentes condições de atmosfera controlada ${ }^{(2,5-9)}$.

Em trabalho recente, Souza e Pardini ${ }^{(10)}$ demonstraram uma relação direta entre o domínio óptico dos planos basais de grafites policristalinos industriais extrudados, calculado via técnicas de microscopia óptica (MO) e pelo comportamento do COF, trabalhando com pares tribológicos semelhantes (contato entre pares do mesmo material). No estudo foi observado um aumento gradual do COF que acompanhou o aumento da área do domínio do plano basal paralelo à superfície de contato. O presente trabalho avalia o comportamento tribológico dos mesmos grafites estudados por Souza e Pardini ${ }^{(10)}$, formando pares tribológicos com aço 4340 , em ambiente controlado a $20{ }^{\circ} \mathrm{C}$ e $50 \%$ UR. Foi correlacionada a predominância do plano basal, identificada por meio de análises de microscopia óptica com luz polarizada (MOLP), ao comportamento tribológico, mensurado por ensaios em tribômetro de pino sobre disco, pela determinação do COF.

\section{Domínio Óptico dos Grafites}

A unidade básica estrutural de um material grafítico é sua célula unitária, conforme mostrado na Fig. 3. Os materiais de carbono são geralmente observados por meio de reflexão com um microscópio óptico, devido a seus altos coeficientes de absorção em comprimentos de onda visíveis ${ }^{(11)}$. Os materiais de carbono, tais como o grafite, opticamente ativos, apresentam superfícies visualizadas em tons de cores variando entre azul, amarelo e rosa, quando observados em luz polarizada ${ }^{(11)}$.

No caso do grafite, esse comportamento permite a verificação do arranjo dos planos, por meio de análise que consiste em isolamento das áreas de interesse e cálculo percentual relativo sobre a área total da amostra. Assim, as cores azul e amarela estão associadas à orientação das arestas do plano basal orientadas a $\pm 45^{\circ}$ em relação à superfície polida da amostra. A coloração rosa está associada à orientação perpendicular ao plano basal à luz incidente do microscópio, ou seja, o plano basal encontra-se paralelo à superfície polida, conforme mostrado na Fig. $4^{(12)}$.

A Fig. 5 ilustra a visualização superficial dos planos de um grafite antes e após a separação, obtida por meio de tratamento computadorizado da imagem, utilizando software Image ${ }^{(10)}$.

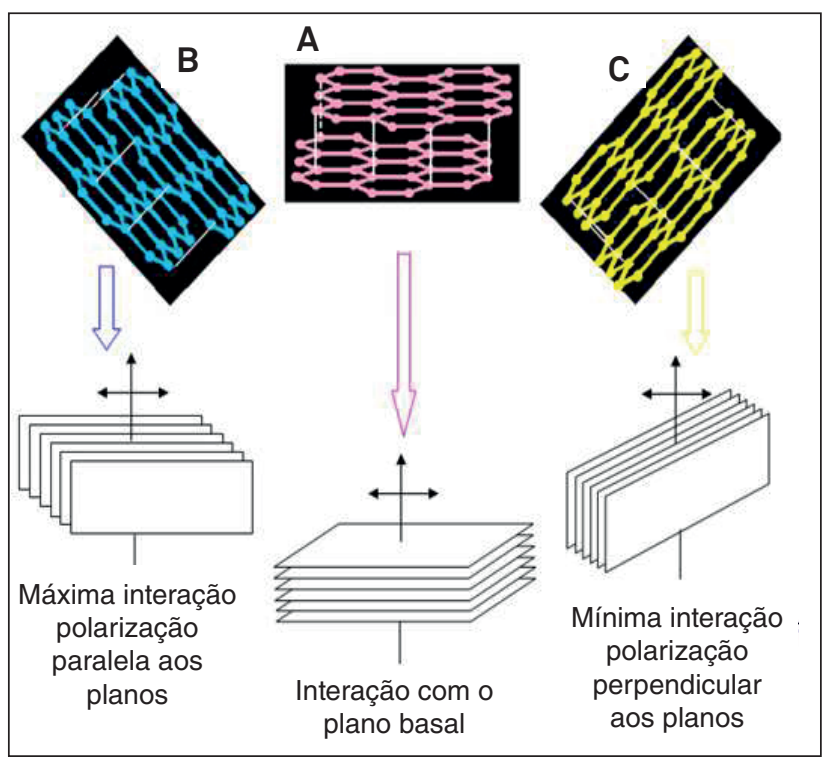

Figura 4: (A) Representação esquemática das diferentes cores observadas em grafites por MOLP. (B) Planos basais perpendiculares à luz incidente. (C) Planos basais posicionados $a \pm 45^{\circ}$ em relação à luz incidente ${ }^{(12)}$.

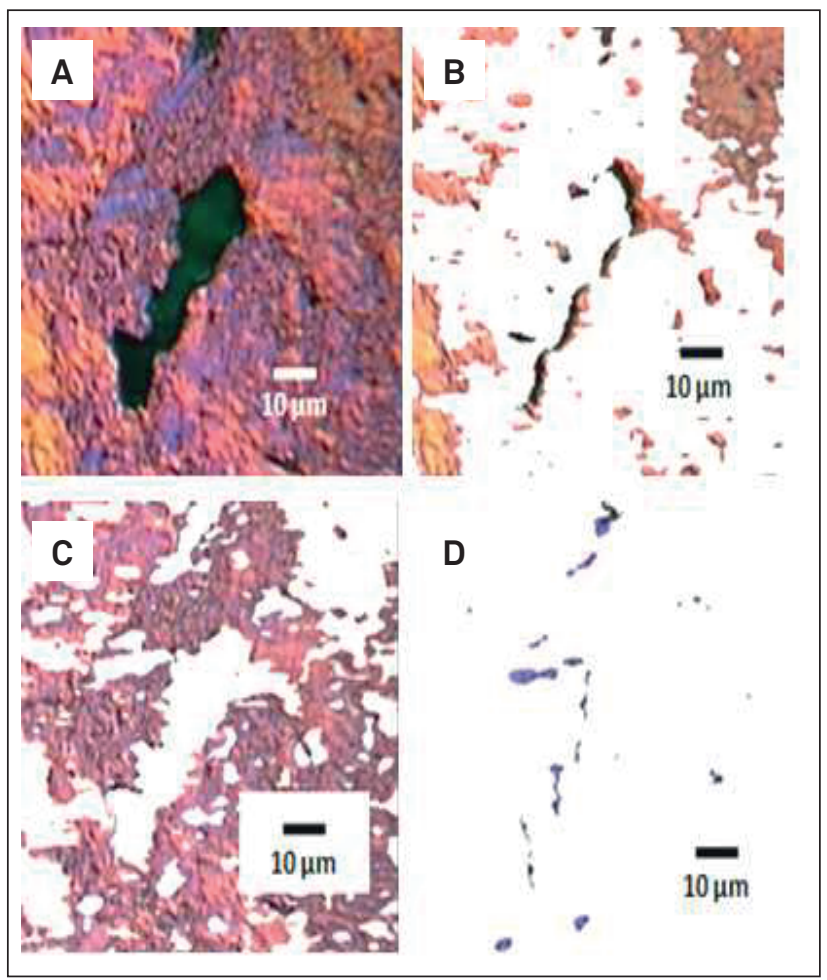

Figura 5: Domínio óptico de um grafite. (A) Superfície sem separação de planos. (B) Plano basal paralelo à superfície da amostra (rosa). (C) e (D) Plano basal com incidência de luz a $\pm 45^{\circ}$ vértices (amarelo e azul) $)^{(10)}$. 


\section{MATERIAIS E MÉTODOS}

\section{Materiais}

Neste trabalho, foi utilizada 1 amostra de aço 4340 e 3 amostras de grafites comerciais policristalinos. Na Tabela 1, é apresentada a caracterização do aço 4340. Os materiais foram fornecidos para teste em tarugos de $50 \mathrm{~mm}$ de diâmetro.

Tabela 1: Especificação técnica do aço 4340.

\begin{tabular}{c|c|}
\hline Propriedade & Valores típicos \\
\hline Resistência a tração (MPa) & $930-1100$ \\
\hline Limite de elasticidade (MPa) & $800-950$ \\
Dureza (HB) & $280-340$ \\
Alongamento (\%) & $10-18$ \\
\hline
\end{tabular}

Foram utilizadas 2 amostras de grafite oriundas da SGL Carbon Group (HLM-85 e HLS). Uma outra amostra de grafite foi manufaturada pela TOKAY Carbon, FE250. Esses materiais passaram a ser identificados, respectivamente, como HLM, HLS e FE. Suas propriedades, fornecidas pelos fabricantes e baseadas no trabalho de Souza e Pardini( ${ }^{(10)}$, são mostradas na Tabela 2.

Tabela 2: Especificação técnica dos grafites.

\begin{tabular}{|c|c|c|c|}
\hline Propriedade & HLM & HLS & FE \\
\hline Massa específica $\left(\mathrm{g} \cdot \mathrm{cm}^{-3}\right)$ & 1,75 & 1,81 & 1,75 \\
\hline Dureza Rockwell $(\mathrm{R})$ & 86 & $105^{*}$ & $82^{*}$ \\
\hline Tamanho do grão $(\mathrm{mm})$ & $\leq 0,8$ & $\leq 0,4$ & $\leq 0,8$ \\
\hline $\begin{array}{c}\text { Módulo elástico (GPa) } \\
\text { Plano basal perpendicular à }\end{array}$ & 11,0 & 15,9 & - \\
\hline superfície (rosa)* & $64,5^{*}$ & $80,9^{*}$ & $55,1^{*}$ \\
\hline
\end{tabular}

*Souza, Pardini(10)

\section{METODOLOGIA}

\section{Ensaio de pino sobre disco}

O ensaio de pino sobre disco foi desenvolvido de acordo com a norma ASTM G 99-05 (Standard Test Method for Wear Testing with a Pin-on-Disk Apparatus) ${ }^{(13)}$, que, entre outros meios, possibilita a avaliação do comportamento tribológico pela avaliação do COF. O tribômetro utilizado é do tipo pino sobre disco, conforme representado na Fig. $6^{(10)}$.

O braço-suporte é acoplado a uma célula de carga que, por meio de um controlador digital, capta os dados da força normal aplicada e da força de atrito gerada entre o par na área de contato. Desta forma, é possível calcular o COF pela Eq. 1.

$$
\mathrm{COF}=\mathrm{Fat} / \mathrm{Fn}
$$

onde: Fat representa a força de atrito; Fn representa a força normal.

As condições complementares aplicadas ao ensaio foram: velocidade tangencial aplicada ao berço rotativo igual a $0,34 \mathrm{~m} \cdot \mathrm{s}^{-1}$, a uma carga de $38,8 \mathrm{~N}$ e distância em que o par é mantido em contato durante a rodagem de $200 \mathrm{~m}$. As condições de ensaio foram selecionadas respeitando-se as limitações operacionais impostas pelo tribômetro, pois a norma não impõe condições específicas ao ensaio. Na Fig. 7, são mostrados os dimensionais aplicados ao pino e ao disco.

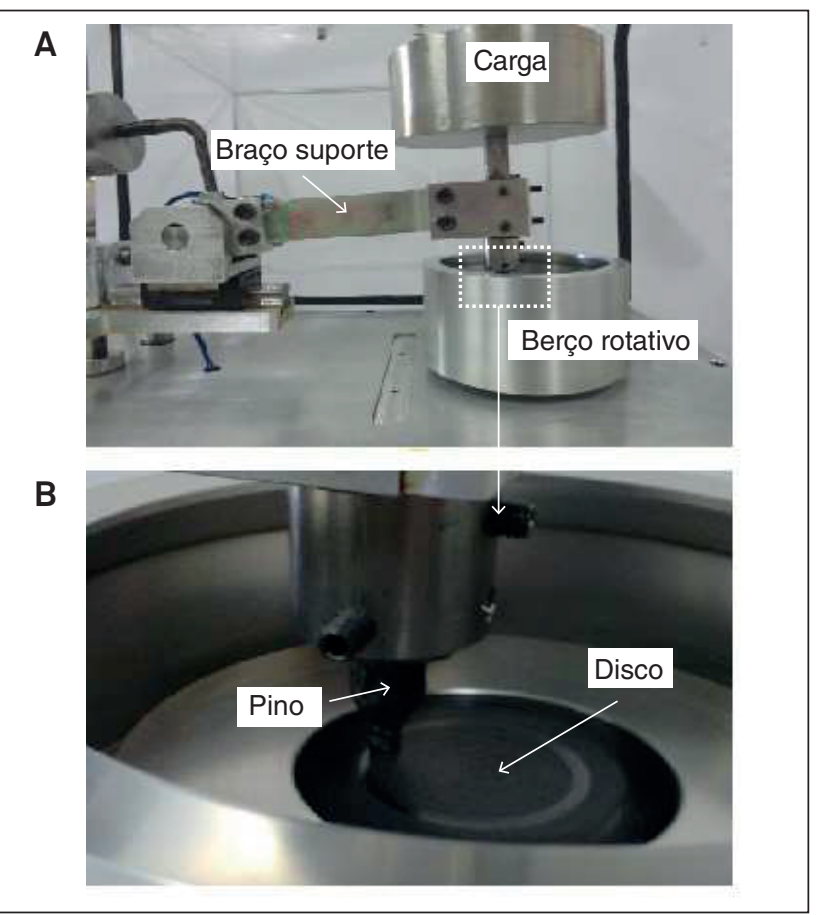

Figura 6: (A) Visão geral do tribômetro de pino sobre disco. (B) Detalhe da área de contato entre pino e disco(10).

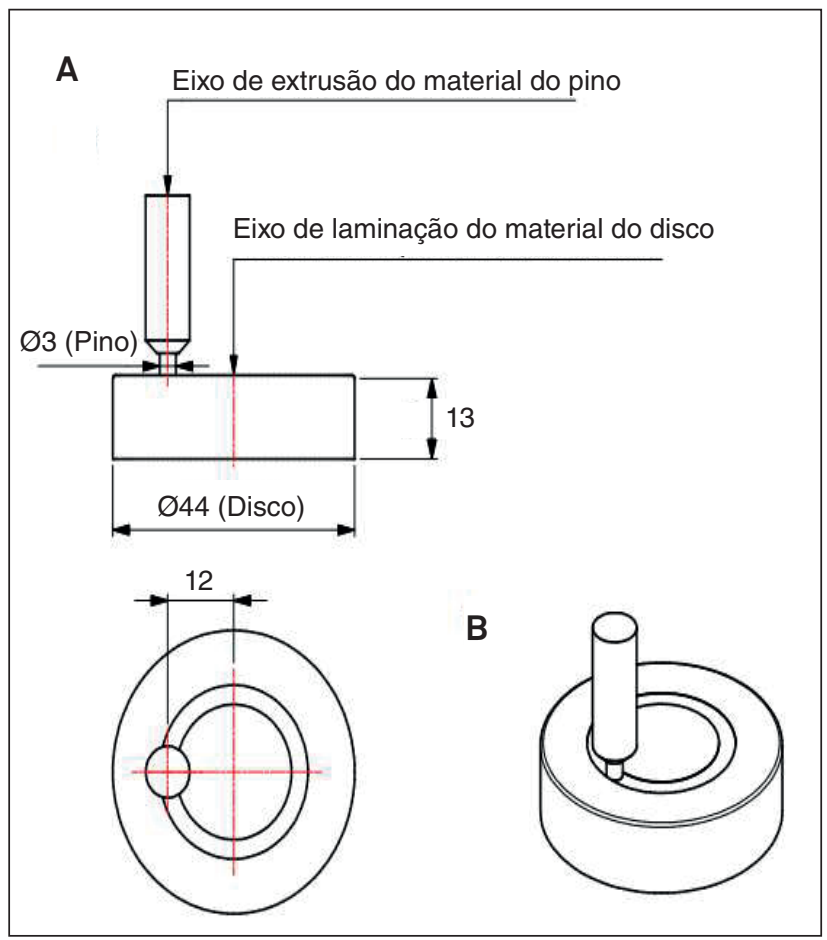

Figura 7: (A) Dimensional ( $\mathrm{mm}$ ) dos pares pino e disco, utilizados no ensaio. (B) Imagem de um disco e de um pino pós-usinagem. 
As amostras de grafite e do aço foram usinados a partir de tarugos de $50 \mathrm{~mm}$ de diâmetro. Tanto o pino quanto o disco foram usinados de tal forma que as superfícies das faces de contato estivessem sempre no sentido do eixo de fabricação dos mesmos, conforme ilustrado na Fig. 7.

Para a montagem dos pares, foi convencionada a confecção da pista em aço e os pinos em grafite, originando-se 3 pares montados de acordo com o exposto na Tabela 3.

Tabela 3: Pares tribológicos utilizados no ensaio.

\begin{tabular}{|c|c|c|}
\hline Par & Pino & Disco \\
\hline 1 & HLM & \\
\hline 2 & HLS & Aço 4340 \\
\hline 3 & FE & \\
\hline
\end{tabular}

\section{Preparação dos Corpos de Prova}

O comportamento tribológico é um fenômeno de superfície, sendo que a rugosidade superficial atua como fator de influência direta no mecanismo de desgaste $\mathrm{e}^{(14,15)}$.

Para a realização dos ensaios tribológicos, foi realizada usinagem dos corpos de prova a partir de corte, torneamento, lixamento e polimento, buscando-se atingir as condições de rugosidade superficial ( $\mathrm{Ra}$ ) recomendadas pela norma ASTM G-99 $(\mathrm{Ra}>0,8 \mu \mathrm{m})^{(13)}$. A Ra foi controlada utilizando-se um rugosímetro da marca Mitutoyo, modelo SJ-301.

\section{RESULTADOS E DISCUSSÃO}

Na Fig. 8, é mostrado um par pino e disco após realização do ensaio.

A Fig. 9 ilustra o comportamento do COF em função da distância de contato percorrida pelos pares tribológicos identificados na Tabela 3, considerando-se os primeiros $2 \mathrm{~m}$ de deslizamento.

Os pontos de referência indicados no gráfico da Fig. 9 limitam 2 fases no comportamento dos pares tribológicos no decorrer do ensaio. Na primeira fase, no início do movimento, ocorre o rompimento da força estática, e são formados os primeiros debris. Nesse estágio, o par tribológico, com o pino FE, possui comportamento destacado dos demais pares devido à rápida formação do filme lubrificante, notada pela rápida estabilização

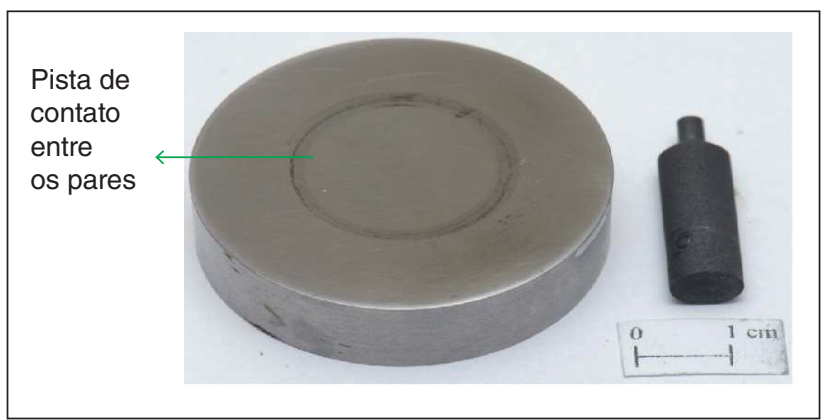

Figura 8: Pino e disco após realização do ensaio. da curva do COF, ocorrida em $\sim 0,15 \mathrm{~m}$, contra $\sim 0,5 \mathrm{~m}$ dos pinos HLS e HLM.

Na segunda fase, ocorre a estabilização do COF dinâmico, representada pelas curvas que tomam forma linear, com pequenos picos de oscilação, caracterizando a formação dos filmes de lubrificação sobre a pista de aço.

A Fig. 10 ilustra o comportamento completo do COF dos pares ensaiados e a Tabela 4 apresenta os resultados médios de COF calculados na fase de estabilização.

O par contendo o pino HLS apresentou o maior valor médio de COF, assim como o maior desvio. O HLS é o grafite que apresentou maior domínio do plano basal perpendicular à face de contato dos componentes do par, 80,9 contra $64,5 \%$ e $55,1 \%$ para o grafite HLM e FE, respectivamente ${ }^{(8)}$. O grafite FE apresentou menores valores médios e mínimos de COF, associado ao menor

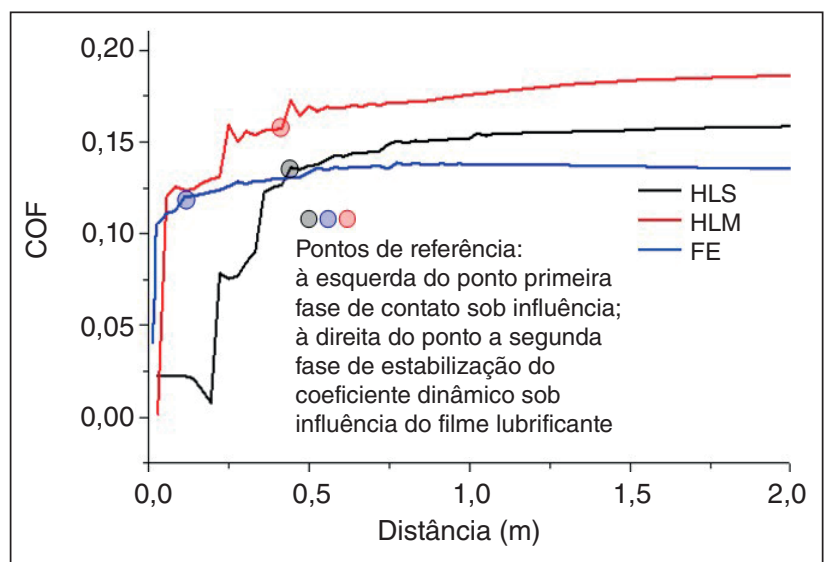

Figura 9: Comportamento do COF em função da distância de deslizamento nos $2 \mathrm{~m}$ iniciais do ensaio tribométrico.

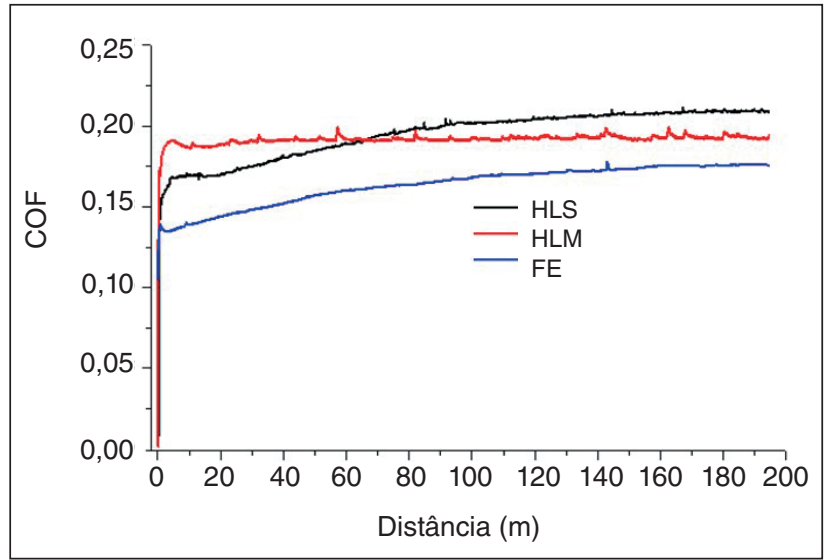

Figura 10: Comportamento do COF em função da distância de deslizamento ao longo dos $200 \mathrm{~m}$ de deslizamento.

Tabela 4: Resultados de COF para os pares ensaios no tribômetro de pino sobre disco, identificados pelo pino em uso.

\begin{tabular}{c|c}
\hline Pino & COF \\
\hline HLM & $0,192 \pm 0,002$ \\
\hline HLS & $0,196 \pm 0,015$ \\
FE & $0,163 \pm 0,011$ \\
\hline
\end{tabular}


valor de domínio do plano basal perpendicular ao movimento. Os valores mais elevados de COF para o grafite HLS (módulo $15,9 \mathrm{GPa}$ ) podem ser também aliados à baixa plasticidade de seus debris frente ao HLM (módulo 11,0 GPa), demonstrando maior eficiência na formação do filme lubrificante do HLM. Isso resulta em menor COF na evolução do contato entre pares.

\section{CONCLUSÕES}

Os resultados mostraram diferenças entre os pares friccionais, o que pode ser explicado considerando-se a evolução do desgaste sobre o plano basal paralelo à superfície de atrito e perpendicular ao movimento de atrito, que é predominante na amostra HLS. Esse fato contribui para um maior poder de adesão, entre pino e disco, a partir da evolução dos danos gerados às ligações desses planos com consequente aumento de $20 \%$ no COF (de 0,163 para 0,196).

A plasticidade dos resíduos formados durante o desgaste dos pares tribológicos influenciam diretamente no comportamento do filme lubrificante, o que pode ser analisado por meio da dureza ou do módulo elástico do material.

\section{AGRADECIMENTOS}

Os autores agradecem ao Centro de Competência em Manufatura (CCM) do Instituto Tecnológico de Aeronáutica (ITA) pela disponibilização do tribômetro de pino sobre disco para a realização deste trabalho.

\section{REFERÊNCIAS}

1. ZUM-GAHR KH. Classification of wear processes. In: Zum-Gahr, $\mathrm{KH}$, editor. Microstructure and wear of materials. N. 10, Tribology Series. Amsterdam: Elsevier; 1987. p. 80-131.

2. CZICHOS H, KLAFFKE D, SANTNER E, WOYDT M. Advances in tribology: the materials point of view. Wear. 1995;190(2):155-61. doi: 10.1016/0043-1648(96)80014-7
3. ORTHWEIN WC. Clutches and brakes desing and selection. Vol. 168, Mechanical engineering. 2nd ed. New York: Marcel Dekker 2004.

4. DRESSELHAUS MS, DRESSELHAUS G, EKLUND PC. Carbon materials. In: DRESSELHAUS MS, DRESSELHAUS G, EKLUND PC. Science of fullerenes and carbon nanotubes. San Diego: Academic Press; 1996. p. 15-30

5. LANCASTERJK. Areview of the influence of environmental humidity and water on friction, lubrication and wear. Tribology International. 1990;23(6):371-89. doi: 10.1016/0301-679X(90)90053-R

6. FOUQUET S, ROLLIN M, PAILLER R, BOURRAT X. Tribological behavior of composites made of carbon fibres and ceramic matrix in the Si-C system. Wear. 2008;264(9-10):850-6. doi: 10.1016/j.wear.2006.12.08

7. YU S, ZHANG F, XIONG X, LI Y, TANG N, KOIZUMI Y, CHIBA A Tribological properties of carbon/carbon composites with various pyrolytic carbon microstructures. Wear. 2013;304(1-2):103-108. doi: 10.1016/j.wear.2013.04.031

8. SCHMITT M, BISTAC S, JRADI K. Tribological behaviour of graphite powders at nano- and macroscopic scales. J Phys Conf Ser. 2007;61(61):1032-6. doi: 10.1088/1742-6596/61/1/204

9. BERMAN D, ERDEMIR A, SUMANT AV. Graphene: a new emerging lubricant. Mater Today. 2014;17(1):31-42. doi: 10.1016/j.mattod.2013.12.003

10. SOUZA MAM, PARDINI LC. Influence of basal plane domains in the tribological behavior of graphites. Matéria (Rio J.). 2016;21(3):706-13. Doi: 10.1590/S1517-707620160003.0067

11. SAVAGE G. Carbon-carbon composites. London: Chapman \& Hall; 1993

12. VON DOLLINGER CFA, PARDINI LC. Optical characterization of polycristaline graphites by image analisys. Anais do $5^{\circ}$ Congresso Brasileiro de Carbono. Vol. 1; 2011; Rio de Janeiro, Brasil. Rio de Janeiro: ABCarb; 2011

13. ASTM, 2010. ASTM G99-05: standard test method for wear testing with a pin-on-disk apparatus. West Conshohocken: ASTM; 2010.

14. BAYER RG. Design for wear resistance. In: ASTM Handbook. Vol 20, Material Selection and Design; 1997. p. 603-14

15. JAHN B, WITTEN E. Composites market report 2013: market developments, trends, challenges and opportunities. Frankfurt: AVK; 2013 